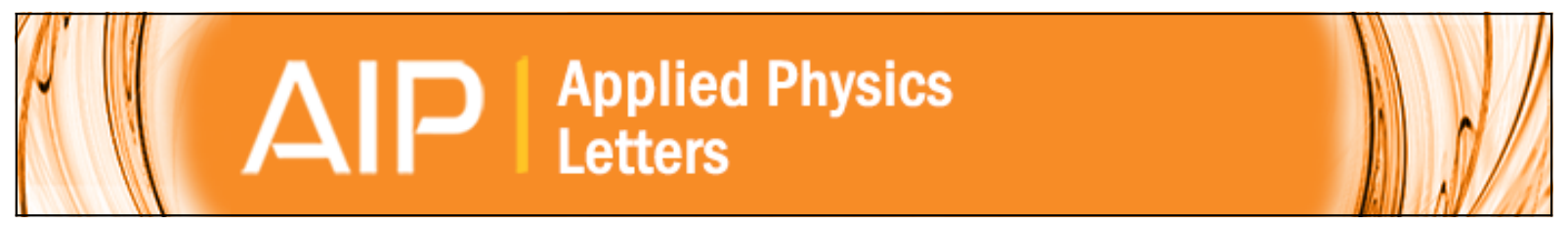

\title{
High-coercivity ultralight transparent magnets
}

M. Gich, LI. Casas, A. Roig, E. Molins, J. Sort, S. Suriñach, M. D. Baró, J. S. Muñoz, L. Morellon, M. R. Ibarra, and J. Nogués

Citation: Applied Physics Letters 82, 4307 (2003); doi: 10.1063/1.1578538

View online: http://dx.doi.org/10.1063/1.1578538

View Table of Contents: http://scitation.aip.org/content/aip/journal/apl/82/24?ver=pdfcov

Published by the AIP Publishing

\section{AlP Re-register for Table of Content Alerts}

\section{Create a profile. 困 Sign up today!}




\title{
High-coercivity ultralight transparent magnets
}

\author{
M. Gich, ${ }^{\text {a) }}$ LI. Casas, A. Roig, and E. Molins \\ Institut de Ciència de Materials de Barcelona (ICMAB-CSIC), Campus UAB, 08193, Bellaterra, \\ Catalunya, Spain \\ J. Sort, S. Suriñach, M. D. Baró, and J. S. Muñoz \\ Departament de Física, Universitat Autònoma de Barcelona, 08193 Bellaterra, Catalunya, Spain \\ L. Morellon and M. R. Ibarra \\ Departamento de Física de la Materia Condensada-ICMA, Universidad de Zaragoza-CSIC, \\ 50009 Zaragoza, Spain \\ J. Nogués \\ Institució Catalana de Recerca i Estudis Avançats (ICREA) and Departament de Física, Universitat \\ Autònoma de Barcelona, 08193 Bellaterra, Catalunya, Spain
}

(Received 27 January 2003; accepted 27 March 2003)

\begin{abstract}
Magnetic silica-aerogel composites have been synthesized by dispersing hard magnetic $\mathrm{Nd}_{2} \mathrm{Fe}_{14} \mathrm{~B}$ particles in a sol during a fast sol-gel process and subsequently supercritically drying the resulting gels. The composites are found to retain most of the outstanding properties of their constituents: the large coercivity and moderate remanence of the magnetic powders and the transparency and low density of silica aerogels. Moreover, aerogels synthesized in the presence of a magnetic field exhibit the alignment of the particles, forming needle-like structures along the direction of the applied magnetic field, which results in optical and magnetic anisotropies. Due to their unique combination of properties, these types of materials may be appealing for magneto-optics and magnetic actuator applications. (C) 2003 American Institute of Physics. [DOI: 10.1063/1.1578538]
\end{abstract}

Aerogels are highly porous solid materials with unusually low densities and high specific surface areas. They are usually prepared by the supercritical drying of highly crosslinked organic or inorganic gels. ${ }^{1}$ Gels derived from the condensation and hydrolysis of metal alkoxide precursors allow the production of a large variety of aerogels, from which silica aerogels have been extensively investigated. Silica aerogels are transparent materials with low-refractive index and low dielectric constant. Thus, they have been proposed for a variety of applications such as optical, thermal, acoustic and electronic materials. ${ }^{2-6}$ The potential applications of silica aerogels have readily increased with the production of composites, covering fields such as catalysis or photoluminescence.$^{7-9}$ Composite aerogels mainly consist in homogeneous dispersions of particles hosted in an aerogel matrix. Aerogels with particles in the nanometer range can be obtained during the gel synthesis from precursors with alkoxide functionalities, since the aerogel nanometric pores limit the particle growth. ${ }^{10}$ However, composite aerogels can also be prepared from nonbonding solid particles added to the sol during the gelation. ${ }^{11}$ In particular, magnetic aerogels have been prepared using both approaches. ${ }^{12-17}$ Most of these magnetic aerogel composites are formed from soft magnetic particles homogeneously dispersed in the aerogel matrix. Consequently, many of the studied systems exhibit low remanence and coercivity at room temperature, thus limiting their possible applications. ${ }^{16}$ Moreover, it has been recently shown that anisotropic distributions of magnetic particles in aerogel matrices can be prepared by using a

${ }^{a}$ Electronic mail: mgich@icmab.es magnetic field during the gelation process. ${ }^{17}$ Nevertheless, even in aligned aerogels, the room temperature magnetic properties remain poor. Taking into account that magnetic aerogels combine the intrinsic properties of the embedded magnetic particles with the superior properties (e.g., low density, transparency, and electrically insulator) of aerogels, it would be interesting for certain applications (e.g., magneto-optical modulation or magneto-optical displays, microwave absorbers, isolators, or modulators) ${ }^{18}$ to have composites of high remanence and high coercivity. In this letter we demonstrate the feasibility to fabricate homogeneous and aligned magnetic aerogel composites containing $\mathrm{Nd}_{2} \mathrm{Fe}_{14} \mathrm{~B}$ particles, which result in ultralight transparent magnetic materials with large coercivities and moderate remanence.

Silica composite aerogels were obtained by supercritical drying of gels prepared by adding different amounts of commercial $\mathrm{Nd}_{2} \mathrm{Fe}_{14} \mathrm{~B}$ particles in a viscous sol. For the gel synthesis, $6.7 \mathrm{ml}$ of a sol consisting of a water solution of ammonia plus a mixture of methanol and tetramethoxysilane (TMOS) was poured into $1 \mathrm{~cm}$ diameter polystyrene test tubes. The molar ratios of the reactants were TMOS: methanol: water:ammonia $=1: 12.25: 4: 6.5 \times 10^{-2}$. This chemical route yields the gelation of the mixture in about 3-4 min. After the addition of the water and the catalyst $\left(\mathrm{NH}_{3}\right)$ the sol was softly stirred and $\mathrm{NdFeB}$ particles, with sizes $\Phi<5 \mu \mathrm{m}$, were added to the viscous solution. During the gelation process, to obtain a gel with a homogeneous dispersion of particles, the test tubes were closed and repeatedly reversed every $4-5 \mathrm{~s}$. Following this procedure three composites A, B, and C containing respectively 50, 300, and $500 \mathrm{mg}$ of $\mathrm{NdFeB}$ powders diluted in $6.7 \mathrm{ml}$ of sol were prepared in order to obtain composites of different magnetic 


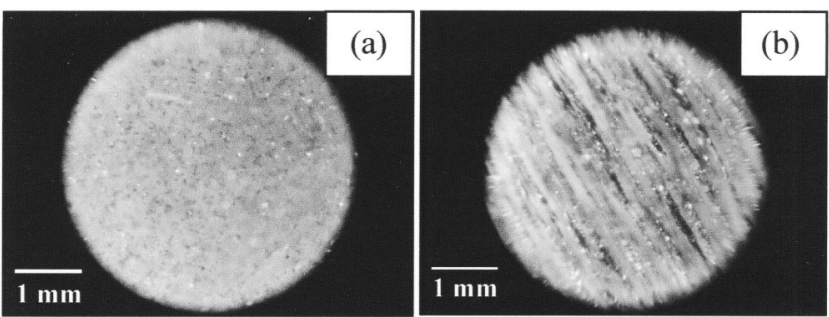

FIG. 1. Photographs of samples B (a) and $\mathrm{B}_{f}$ (b) using a $50 \times$ magnification.

phase concentrations. Furthermore, aiming to investigate the effects of in-field synthesis, additional samples $\left(\mathrm{A}_{f}, \mathrm{~B}_{f}\right.$, and $\mathrm{C}_{f}$ ) of the same compositions and prepared following the same procedure were placed in an $8 \mathrm{kOe}$ homogeneous magnetic field when the viscosity was so high that no particle sedimentation was observed. The direction of the magnetic field, was orthogonal to the test tube axis and the samples were kept under field for $5 \mathrm{~h}$. The gels were then removed from their tubes and aged in a methanol solution at room temperature for 10 days. The central and more homogeneous parts of gel monoliths were selected and supercritically dried. In this process the gel solvent (methanol) was exchanged by liquid $\mathrm{CO}_{2}$ at 100 bar and $23^{\circ} \mathrm{C}$. After the solvent exchange, pressure and temperature were set over the $\mathrm{CO}_{2}$ critical point $\left(72.9\right.$ bar and $\left.31{ }^{\circ} \mathrm{C}\right)$, where the liquid $\mathrm{CO}_{2}$ filling the pores completely transformed to gas. Finally, the pressure was decreased keeping the gel structure with $\mathrm{CO}_{2}$ gas inside the pores that after opening the high pressure plant were filled with air. A supercritical drying with methanol was attempted at $239.45^{\circ} \mathrm{C}$ but resulted in a deterioration of the composite magnetic properties due to the thermallyinduced structural changes in the $\mathrm{Nd}_{2} \mathrm{Fe}_{14} \mathrm{~B}$ particles.

The obtained $\mathrm{NdFeB}$ composite aerogels are cylindrical monoliths. The appearance of the samples depends on the content of magnetic particles and on whether they have been synthesized in-field or without field. It is observed that in the aerogels synthesized in the presence of a magnetic field the magnetic particles are aligned forming needle-like structures along the direction of the magnetic field applied during the gelation process. This indicates that when the gels were placed under the magnetic field, the particles were still able to slightly move and consequently align following the field lines. In contrast, the aerogels prepared in absence of a magnetic field keep the relatively homogeneous suspension of magnetic particles from the gelation. This can be clearly observed in Fig. 1 Moreover, the transparency of the composites decreases when the $\mathrm{NdFeB}$ content increases. In fact, only the monoliths of samples $\mathrm{A}$ and $\mathrm{A}_{f}$, having the lowest powder content, are found to be transparent in all the directions across the cylinder axis. However, for the in-field aerogels the transparency increases along the particle alignment direction and decreases in the direction perpendicular to it (see Fig. 2). Samples $\mathrm{B}_{f}$ and $\mathrm{C}_{f}$ exhibit a transparency similar to that of A when the light is applied parallel to the orientation direction, although the density of particles is 6 and 10 times larger, respectively. However, when the light is perpendicular to the alignment direction samples $\mathrm{B}_{f}$ and $\mathrm{C}_{f}$ appear opaque. The anisotropic transparency presented by the in-field composites could make them suitable for certain applications in magnetooptics (e.g., based on dichroism or

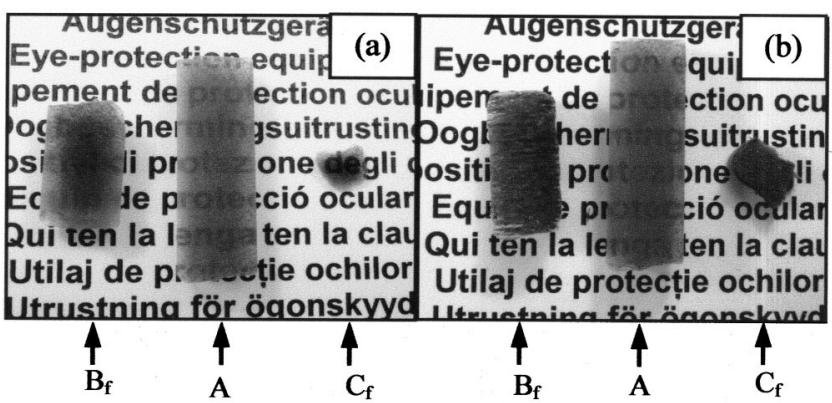

FIG. 2. Photographs of monoliths of samples $\mathrm{B}_{f}, \mathrm{~A}$, and a piece of sample $\mathrm{C}_{f}$. The in-field synthesized samples $\left(\mathrm{B}_{f}\right.$ and $\left.\mathrm{C}_{f}\right)$ are placed so that the direction of particle alignment is perpendicular (a) and parallel to the photograph plane (b).

birefringence), which have been largely investigated in other magnetically anisotropic systems. ${ }^{19}$

As can be seen in Table I, the in-field composites exhibit systematically a larger density than the homogenous ones. This effect can be explained by the particles tendency during the gelation to accumulate close to the center of the gel where the magnetic field is higher.

Surface area measurements by $\mathrm{N}_{2}$ isotherm adsorption and Brunnauer-Emmet-Teller (BET) analysis were performed (Table I), and they show typical aerogel surface area values, ranging from 490 to $810 \mathrm{~m}^{2} / \mathrm{g}$. The samples with higher powder contents have lower surface areas, as would be expected. The lower surface areas measured for in-field synthesised composites as compared to the samples produced without field can be due to higher presence of pores bigger than $100 \mathrm{~nm}$, not detected by the BET technique. These are probably induced during the synthesis by the displacement of the magnetic particles under the action of the field.

Room temperature hysteresis loops have been performed for all the samples, in fields up to $120 \mathrm{kOe}$, in a vibrating sample magnetometer. The in-field composites were measured with the field applied parallel and perpendicular to the synthesis direction. All composites display the typical features of a hard magnetic phase, i.e., large coercivity, $H_{C}$, and moderate squareness, $M_{R} / M_{S}$, (Fig. 3), thus indicating that the hard magnetic properties of the $\mathrm{NdFeB}$ powders have not been altered during the composite aerogel synthesis. All the studied aerogels exhibit coercivities in the range of $H_{C}$ $\sim 13 \mathrm{kOe}$, i.e., well within the range of coercivities characteristic of melt spun $\mathrm{Nd}_{2} \mathrm{Fe}_{14} \mathrm{~B}^{20}$ and squareness values just above $M_{R} / M_{S}=0.5$ that are typical of isotropic magnets with exchange interactions. ${ }^{21}$ The effect of particle alignment is evidenced in the difference of the values of $M_{R} / M_{S}$ be-

TABLE I. Bulk density and surface area of magnetic aerogel composites synthesized in-field and without field with different amounts of $\mathrm{Nd}_{2} \mathrm{Fe}_{14} \mathrm{~B}$ powders.

\begin{tabular}{|c|c|c|c|}
\hline Sample & $\begin{array}{l}\mathrm{NdFeB} \text { content } \\
(\mathrm{mg})\end{array}$ & $\begin{array}{c}\text { Bulk density } \\
\left(\mathrm{g} / \mathrm{cm}^{3}\right)\end{array}$ & $\begin{array}{l}\text { Surface area } \\
\left(\mathrm{m}^{2} / \mathrm{g}\right)\end{array}$ \\
\hline A & 50 & 0.14 & 810 \\
\hline $\mathrm{A}_{f}$ & 50 & 0.14 & 790 \\
\hline B & 300 & 0.15 & 760 \\
\hline $\mathrm{B}_{f}$ & 300 & 0.18 & 670 \\
\hline $\mathrm{C}$ & 500 & 0.20 & 650 \\
\hline$C_{h e}$ & t: 500 & 0.22 & 490 \\
\hline
\end{tabular}




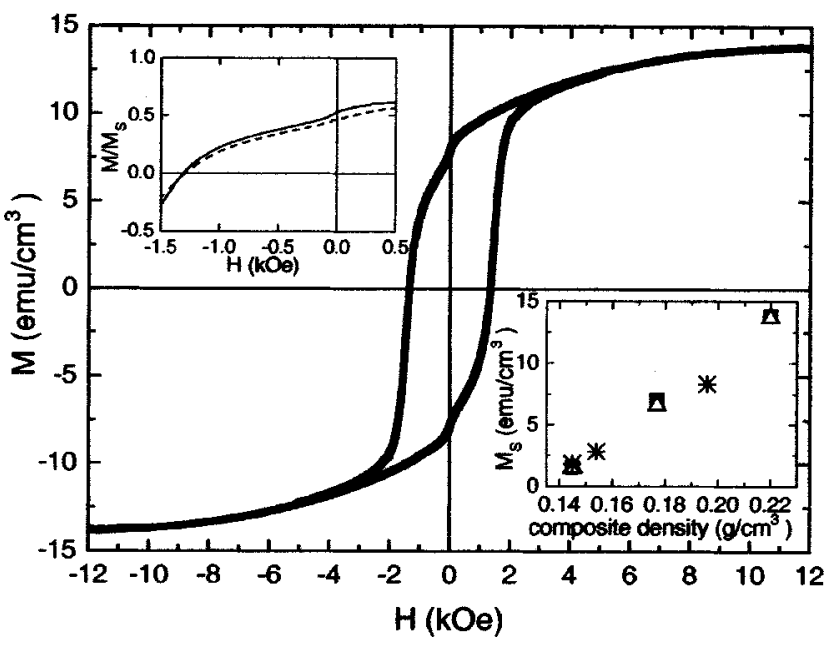

FIG. 3. Hysteresis loop for sample $\mathrm{C}_{f}$ with the applied field parallel to the particle alignment direction. The inset shows the saturation magnetization, $M_{S}$, for (ם) in-field samples with the field applied at $0^{\circ}$ from the synthesis direction, $(\triangle)$ in-field samples with the field applied at $90^{\circ}$ from the synthesis direction and $(*)$ samples synthesized without field. Shown in the inset is the normalized magnetization for sample $\mathrm{B}_{f}$ along (solid line) and perpendicular to the aligning direction (dashed line).

tween the composites synthesized in-field or without field. For the in-field synthesized samples, when the measuring field was applied at $0^{\circ}$ with respect to the powders alignment direction, $M_{R} / M_{S}=0.58$ was obtained, whereas when the measurements were carried out at $90^{\circ}$ the squareness decreased to $0.53-0.54$ (see inset Fig. 3). In the case of the samples synthesized without field one obtains values of $0.54-0.55$. The small difference between the parallel and perpendicular squareness evidences a certain degree of magnetic alignment. This shows that, besides physical orientation of the particle powders along the field lines, some magnetic anisotropy can be induced in the samples. Note that since the particles of several micron in size are multidomain and composed of several randomly oriented crystallites a full magnetic orientation is impossible in these samples. However, full magnetic and physical alignment should be achievable for single domain particles.

Moreover, the energy product, $(B H)_{\max }$, was estimated from the hysteresis loop, where a value of $0.06 \mathrm{~kJ} / \mathrm{m}^{3}$ was obtained for $\mathrm{C}_{f}$. Yet, typical $B H_{\max }$ values for $\mathrm{NdFeB}$ powders are of the order of $100 \mathrm{~kJ} / \mathrm{m}^{3}$, about 1700 times higher. This difference can be understood on the basis of the linear dependence of the saturation magnetization, $M_{S}$, with the composite bulk density (see inset of Fig. 3) and the fact that $B H_{\max }$ scales with $M_{s}^{2}$; keeping the magnetic powders content constant and reducing the sample density by a factor of about 40 (from $7.8 \mathrm{~g} / \mathrm{cm}^{3}$ of the $\mathrm{NdFeB}$ powders to the $0.2 \mathrm{~g} / \mathrm{cm}^{3}$ of the composite $\mathrm{C}_{f}$ ), can explain a lowering of the $B H_{\max }$ by a factor of 1600 , in agreement with our experimental values.

Sample $\mathrm{C}_{f}$ shows the highest remanence of the studied composite aerogels, which is around $M_{R} \sim 8 \mathrm{emu} / \mathrm{cm}^{3}$ (i.e., $\left.4 \pi M_{R} \sim 100 \mathrm{G}\right)$. The fields at the surface of the composite aerogel monoliths, after magnetization in a pulsed field of about $30 \mathrm{kOe}$, were measured using a gaussmeter. Samples $\mathrm{C}_{f}$ and $\mathrm{B}_{f}$ gave values in excess of 40 and $15 \mathrm{Oe}$, respectively. Even though the magnetic fields created by the magnetic aerogels composites can be considered somewhat small, they are sufficient to attract small stainless steel pieces (e.g., needles). Moreover, in spite of their low remanence and due to their low density, these aerogels are rather sensitive, e.g., they easily move or they give magnetomechanical response, even to very small fields.

In conclusion, we have demonstrated that it is possible to fabricate homogeneous and aligned magnetic aerogel composites from $\mathrm{Nd}_{2} \mathrm{Fe}_{14} \mathrm{~B}$ particles. The composites exhibit excellent magnetic and aerogel properties, e.g., large coercivity $\left(H_{C} \sim 13 \mathrm{kOe}\right)$, moderate squareness $\left(M_{R} / M_{S} \sim 0.55\right)$, low density $\left(\rho \sim 0.14-0.22 \mathrm{~g} / \mathrm{cm}^{3}\right)$ and transparency. Due to this combination of properties this type of material should be considered as a potential candidate for applications in the fields of magneto-optics and magnetic actuators.

${ }^{1}$ N. Hüsing and U. Schubert, Angew. Chem. 37, 22 (1998).

${ }^{2}$ T. Sumiyoshi, I. Adachi, T. Ijima, R. Suda, M. Yokoyama, and H. Yokogawa, J. Non-Cryst. Solids 225, 369 (1998).

${ }^{3}$ D. Carlson, D. Lewis, K. McKinley, J. Richardson, and T. Tillotson, J. Non-Cryst. Solids 186, 372 (1995).

${ }^{4}$ N. Kawakami, Y. Fukumoto, T. Kinoshita, K. Suzuki, and K. Inoue, Jpn. J. Appl. Phys., Part 2 39, L182 (2000).

${ }^{5}$ T. Burger and J. Fricke, J. Phys. Chem. 102, 1 (1998).

${ }^{6}$ R. Gerlach, O. Kraus, J. Fricke, P. C. Eccardt, and N. Kroemer, J. NonCryst. Solids 145, 227 (1992).

${ }^{7}$ S. H. Lee, D. J. Suh, T. J. Park, and K. L. Kim, Catal. Commun. 3, 441 (2002).

${ }^{8}$ J. Shen, J. Wang, B. Zhou, Z. Deng, Z. Weng, L. Zhu, L. Zhao, and Y. Li, J. Non-Cryst. Solids 225, 315 (1998).

${ }^{9}$ D. D. Smithy, L. A. Snow, L. Sibille, and E. Ignont, J. Non-Cryst. Solids 285, 256 (2001).

${ }^{10}$ A. Chatterjee and D. Chakravorty, J. Phys. D 23, 1097 (1990).

${ }^{11}$ M. L. Anderson, R. M. Stroud, C. A. Morris, C. I. Merzbacher, and D. R. Rolison, Adv. Eng. Mater. 2, 481 (2000).

${ }^{12}$ L. Zhang, G. C. Papaeftthymiou, and J. Y. Ying, J. Appl. Phys. 81, 6892 (1997); R. D. Shull, J. J. Ritter, A. J. Shapiro, L. J. Swartzendruber, and L. H. Bennett, ibid. 67, 4490 (1990).

${ }^{13}$ G. Ennas, M. F. Casula, A. Corrias, and G. Paschina, J. Non-Cryst. Solids 293-295, 1 (2001); A. Yasumori, H. Matsumoto, S. Hayashi, and K. Okada, J. Sol-Gel Sci. Technol. 18, 249 (2000).

${ }^{14}$ C. Savii, M. Popovici, C. Enache, J. Subrt, D. Niznansky, G. Filoti, and M. Morariu, Solid State Ionics 151, 219 (2002).

${ }^{15}$ L1. Casas, A. Roig, E. Molins, J. M. Grenèche, J. Asenjo, and J. Tejada, Appl. Phys. A: Mater. Sci. Process. 74, 591 (2002).

${ }^{16}$ Ll. Casas, A. Roig, E. Rodríguez, E. Molins, J. Tejada, and J. Sort, J. Non-Cryst. Solids 285, 37 (2001).

${ }^{17}$ N. Leventis, I. A. Elder, G. J. Long, and D. R. Rollison, Nano Lett. 2, 63 (2002).

${ }^{18}$ L. L. Beecroft and C. K. Ober, Chem. Mater. 9, 1302 (1997).

${ }^{19}$ S. Y. Yang, Y. P. Chiu, B. Y. Jeang, H. E. Horng, C.-Y. Hong, and H. C. Yang, Appl. Phys. Lett. 79, 2372 (2001).

${ }^{20}$ B. M. Ma, J. Herchenroeder, B. Smith, M. Suda, D. N. Brown, and Z. Chen, J. Magn. Magn. Mater. 239, 418 ( 2002).

${ }^{21}$ R. Fischer, T. Schrefl, H. K. Kronmüller, and J. Fidler, J. Magn. Magn. Mater. 153, 35 (1996). 